\title{
La paradoja de Winnicott: un enfoque filosófico de las nociones de relación y uso de un objeto*
}

\author{
Carlos Caorsi \\ Universidad de la República \\ Montevideo, Uruguay
}

El artículo discute los conceptos "relación de objeto" y "uso del objeto", teniendo en cuenta su sentido y la perspectiva de la primera y tercera persona con respecto a ellos. Para una mejor comprensión del significado de estos conceptos Winnicott propone que se acepten las paradojas que subyacen a éstos; es por ello que se analizan las dicotomías bebé-ambiente y creación-destrucción de un objeto. En la primera se destacan las cualidades objetivas de la madre (preocupación maternal primaria) como relevantes para el desarrollo normal del bebé ya que se produce una dilatación de la gratificación; en la segunda (creación-destrucción de un objeto), se resalta la tensión instintiva del bebé (creatividad primaria) y su relación con la preocupación maternal primaria que origina la creación del objeto, el mismo que es de tipo subjetivo que genera la secuencia evolutiva de la relación de objeto al uso del objeto.

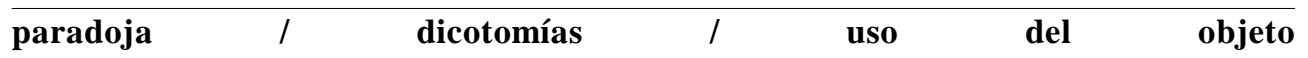

\section{The Winnicott paradox: a philosophical view of the notion of relation and use of the object}

The concepts of "object relation" and " use of an object" are discussed in the article. The author takes into account the perspective of the first and third person in relation to them. For a better understanding of the meaning of these concepts Winnicot propose the acceptance of the two underlying paradoxes; then the dichotomies environment-baby and creation-destruction of an object are analyzed. In the first paradox (environment-baby) the objective qualities of the mother are salient (maternal primary worry) as relevant for the normal development of the baby because there is a dilatation of the gratification. In the second paradox (creation-destruction of an object), the instinctive tension of the baby (primary creativity) is salient and its relationship with the maternal primary worry which generate the object creation that is subjective, that generates the evolutive sequence of the relation from the object relation to the use of an object.

\begin{tabular}{lllllll}
\hline paradox $/$ & dichotomy & $/$ & use & of the object
\end{tabular}

Quedo en deuda con Cristina Barcia, con quien discutí ideas de este trabajo y me aportó observaciones que sirvieron para aclararme algunos puntos de los aquí tratados. 
Los conceptos de "relación de objeto" y "uso de un objeto" pertenecen al tipo de conceptos teóricos que, por su acta de nacimiento, ofrecen no pocas dificultades a todo intento de hacer una precisión filosófica de éstos. No en vano Winnicott nos alerta acerca de que aceptemos las paradojas que subyacen a aquéllos, ya que sólo así podemos llegar a comprender su significación ${ }^{1}$. Son varias las razones que originan esta dificultad.

Por un lado, en la descripción y caracterización que Winnicott hace de dichos conceptos, es necesario tener en cuenta cuando son hechas desde la perspectiva de la tercera persona o de la primera. Así, por ejemplo, cuando habla de la "primera posesión no-yo", refiriéndose a los objetos transicionales, por momentos no sabemos si se trata de no-yo desde el punto de vista del observador (perspectiva de la tercera persona) o de no-yo en lo que respecta a las vivencias del bebé (perspectiva de la primera persona).

Por otro lado, no es claro si todas las veces que utiliza el término "uso" de un objeto lo hace en el mismo sentido o si debemos atribuir sentidos diferentes a esas distintas ocurrencias. Así, por ejemplo, cuando también a propósito

1 "Este paso (de la relación al uso) significa que el sujeto destruye el objeto. Sobre esa base el filósofo de gabinete podría argumentar que por consiguiente no existe, en la práctica, el uso de un objeto: Si éste es exterior, es destruido por el sujeto. Pero si el filósofo abandona el sillón de su gabinete y se sienta en el suelo con su paciente, encontrará que hay una posición intermedia" (Winnicott, 1971; p. 121). de los fenómenos y objetos transicionales dice que de lo que se trata no es del objeto sino del uso del objeto (Winnicott, 1971; p. 14) no es claro si debemos entender el término "uso" en el preciso sentido que pretende otorgarle en el capítulo 6 de la misma obra. En un sentido parecería que no (Winnicott, 1971; p. 18, punto 2) si atendemos a la afirmación según la cual la zona de los fenómenos transicionales es una "zona intermedia de experiencia entre el erotismo oral y la verdadera relación del objeto"; y por otro lado, si tomamos en cuenta la afirmación de que el uso del objeto supone una secuencia que va de la relación de objeto, pasando por la destrucción del objeto, al uso del objeto. Claro que también podría llegarse a la conclusión contraria si se entiende que la cualidad de "verdadera" atribuida a la relación de objeto en el pasaje citado tiene la finalidad de establecer una diferencia con lo que en sentido más estricto llama relación de objeto.

Por cierto que la situación podría no ser del todo desesperante si tomamos a la ligera la conocida afición de Winnicott por las paradojas. Pero eso no es una salida; una cosa son las paradojas y otra nuestras confusiones. De modo que aunque no espero aclarar en su totalidad estas dificultades haré un esfuerzo por arrojar luz, allí donde mis capacidades me lo permitan. Tal vez eso sirva para desbrozar un poco el camino a un intento de clarificación más profundo. 
Con el fin de llevar adelante este ensayo de clarificación conceptual debo detenerme como paso previo en dos dicotomías propuestas por Winnicott y su crítica a una tercera.

Las dos dicotomías propuestas son la que comprende la estructura bebé-ambiente y la de creación y destrucción de un objeto. La dicotomía rechazada por insuficiente es la clásica entre realidad interior o personal y realidad exterior y compartida. Veamos muy someramente cómo éstas se articulan.

\section{BEBÉ-MEDIO AMBIENTE}

La teoría del desarrollo en Winnicott se articula a través de la relación bebémedio ambiente, el cual -en este caso específico- es provisto por la madre.

En este sentido podemos limitar a la madre el medio ambiente relevante para el bebé. Que el bebé sin la madre no puede sobrevivir es una consecuencia de la natural indefensión del infante humano.

Pero lo relevante en el planteamiento de Winnicott es el énfasis que pone en ciertas cualidades objetivas de la madre como imprescindibles para el desarrollo normal del bebé.

Dichas cualidades son las que Winnicott incluye en el concepto de madre lo bastante buena. Y la característica fundamental de ésta es la preocupación maternal primaria.

Destacamos entonces de esta primera dicotomía lo decisivo de ciertas características objetivas del ambiente.

\section{CREación Y DESTRUCCIÓN DEL OBJETO}

La madre es pues quien satisface las necesidades del bebé. Veamos lo que pasa con esas necesidades desde el lado de este último. Dado el marco proporcionado por la madre, el bebé es capaz de concebir la idea de algo que podría satisfacer la creciente necesidad que surge de la tensión instintiva. Esto es lo que Winnicott denomina creatividad primaria. Así, a instancias de la tensión generada por la necesidad, se produce en el niño la idea de que algo va a salir al encuentro de esa necesidad, aun cuando en principio no se pueda decir que el niño sepa lo que va a crear. En ese momento, y en virtud de la preocupación maternal primaria, la madre provee el pecho coincidentemente con la necesidad del bebé. Tal encuentro genera en éste la ilusión de que él ha creado el pecho, el cual se constituye en un objeto subjetivo bajo su control omnipotente. De esta manera la creación del objeto se produce en la intersección de la necesidad del niño y lo aportado por la madre.

\section{RECHAZO DE UNA DICOTOMÍA}

De acuerdo con esta descripción la creación del objeto se produce en una intersección entre lo interno, creatividad primaria, y lo externo, la adaptación de la madre a las necesidades del bebé, la madre lo bastante buena. $\mathrm{Si}$ bien lo que resulta es un objeto subjeti- 
$v o$, no es posible describir este fenómeno ni como puramente subjetivo ni como objetivo. De hecho confluyen para que en éste sea posible tanto lo subjetivo como lo objetivo. Es en ese sentido que Winnicott sostiene la insuficiencia de la dicotomía tradicional subjetivo-objetivo. La descripción de este tipo de fenómenos supone una tercera zona intermedia entre ambas, a la que denominará zona de la ilusión, zona por excelencia de los fenómenos transicionales.

\section{RELACIÓN DE OBJETO Y USO DEL OBJETO}

Hecha esta breve síntesis de algunos de los conceptos winnicottianos que necesitaremos para la clarificación que nos proponemos, podemos entrar de lleno en el tema que nos ocupa.

Lo primero que debemos precisar es de qué estamos hablando cuando usamos los conceptos de "relación de objeto" y "uso del objeto". En un sentido estrictamente formal, y más allá del uso del término "relación" en uno solo de estos conceptos, en ambos casos se trata de una relación. O sea que tanto la "relación de objeto" como "el uso de un objeto" pertenecen a la categoría de las relaciones. Ambas son además relaciones diádicas, es decir, que se dan entre dos individuos. Podemos denominar a esos individuos sujeto y objeto, respectivamente. De acuerdo con esto, tanto la relación de objeto como el uso del objeto son relaciones que se esta- blecen entre sujeto y objeto. Entre los distintos tipos de relaciones que se pueden establecer entre un sujeto y un objeto se encuentran los de "relación de" y "uso del". Las diferencias entre ambas van a estar dadas por el tipo de relación que se da en cada una. Sin embargo, al entrar a considerar esas diferencias no podemos olvidar la perspectiva (de la tercera o primera persona) desde la cual realizamos dichas consideraciones, ya que en algunos casos ambas pueden coincidir desde una perspectiva y diferir desde la otra. Las diferencias que Winnicott considera, podrían caracterizarse desde los siguientes cuatro puntos de vista: 1) desde la identidad de los objetos entre los cuales se dan esas relaciones, 2) desde el control que la relación permite ejercer al sujeto sobre el objeto, 3) desde las modalidades del objeto, y 4) desde la persistencia del objeto.

Veamos cada una de ellas.

\section{Diferencia en cuanto a la identidad de los objetos}

En lo referente a este punto se hace imprescindible precisar la perspectiva desde la cual se establecen las diferencias, ya que, como veremos, los resultados son diferentes en ambos casos.

Así, desde la perspectiva de la tercera persona tanto en el caso de la relación como en el del uso, sujeto y objeto son entidades diferentes. Sin embargo, desde la perspectiva de la primera persona en el caso de la relación 
sujeto y objeto coinciden ${ }^{2}$, en tanto que el del uso sujeto y objeto son diferentes.

\section{Diferencias en cuanto al control del objeto por el sujeto}

Aquí parece que sólo cabe la perspectiva de la primera persona, ya que la experiencia de control es, en el caso que nos ocupa, un fenómeno fundamentalmente subjetivo.

La diferencia estriba en que, en tanto que en la relación de objeto el sujeto controla omnipotente al objeto, en el caso del uso el objeto ha escapado al control omnipotente del sujeto.

Ha sido ubicado, en palabras de Winnicott, fuera de la zona de control omnipotente.

\section{Diferencias en cuanto a las cualidades del objeto}

De lo que se trata es de saber si las cualidades que en la relación se le asignan al objeto son cualidades objetivas de éste o no.

Así, en el caso de la relación de objeto las cualidades asignadas al objeto no son sus cualidades objetivas, sino que son resultado de las proyecciones del sujeto.

2 "Dos bebés se alimentan a pecho. Uno (se refiere al caso de la relación de objeto) se alimenta de la persona, pues el pecho y él todavía no se han convertido (para él) en fenómenos separados" (Winnicott, 1971, p. 120). El intercalado entre paréntesis y el subrayado son nuestros.
El objeto se reduce en este caso a ser un manojo de proyecciones del sujeto ${ }^{3}$. De modo diferente, en el caso del uso las cualidades relevantes del objeto son sus características propias reales.

\section{Diferencias en cuanto a la persistencia del objeto}

En este último caso, las diferencias se establecen en virtud del carácter continuo o discontinuo del objeto. El objeto de la relación es un objeto discontinuo o inconstante en términos de Winnicott.

En efecto, existe en tanto que es creado por el bebé y deja de existir en tanto que es destruido por el mismo. El objeto es así la suma discontinua de todas las creaciones del mismo por el bebé. En cambio, en el caso del uso del objeto, éste ha adquirido una continuidad espacio-temporal resultado de su independencia respecto del bebé y de sus necesidades. El objeto ya no será creado sino que será encontrado.

\section{LA SECUENCIA EVOLUTIVA DE LA}

\section{RELACIÓN DE OBJETO AL USO DEL}

\section{OBJETO}

Hasta el momento nos hemos limitado a consignar las diferencias señala-

3 Habría que precisar que aún en este caso las modalidades del objeto podrían ser las objetivas en el caso en que hubiera una coincidencia entre las cualidades proyectadas y las reales. Sin embargo lo que importa es que es vivido como teniendo esas cualidades porque las mismas le han sido proyectadas y no porque en realidad las posee. 
das por Winnicott entre la relación de objeto y el uso del objeto. Con el fin de aclarar un poco más la diferencia entre ambas nociones será necesario que nos detengamos en la secuencia evolutiva que va de la relación al uso.

Podríamos esquematizar dicha secuencia evolutiva del siguiente modo: relación de objeto-destrucción de objeto-uso del objeto.

Como vemos, la enumeración de la secuencia saca a luz una de las paradojas a las que Winnicott es tan aficionado. Si el objeto ha sido destruido ¿cómo se le puede usar? Por otra parte, coloca en el lugar central la destrucción del objeto, uno de los elementos de la dicotomía que tratamos y que ex profeso nos hemos limitado a mencionar sin decir casi nada acerca de la misma. La razón de esto es que pensábamos detenernos en ella en el desarrollo que sigue.

Pero volvamos a nuestra secuencia evolutiva. Según hemos señalado, tanto la relación de objeto como el uso del objeto son relaciones. Ambas son relaciones que se dan entre un sujeto y un objeto. Siendo así, explicar la referida evolución se reduciría a explicar de qué forma una relación que tiene características de una relación de objeto se transforma en una relación con las características del uso de un objeto. Es decir cómo se va dando el cambio de cualidades que transforma una relación de objeto en el uso de un objeto. De acuerdo con las cualidades diferenciales se- ñaladas entre ambos tipos de relación, esa evolución supone que se produzcan los cuatro cambios siguientes:

1. Paso de la relación con un objeto-yo a un objeto no-yo.

2. Paso de control omnipotente del objeto a la pérdida de este control.

3. Paso de un objeto como manojo de proyecciones a un objeto con cualidades objetivas.

4. Cambio de un objeto discontinuo a un objeto continuo.

Veamos de qué modo la secuencia evolutiva puede dar cuenta de estas modificaciones. Como señalamos, la destrucción del objeto juega un papel central en este proceso. Sin embargo, debemos entender que ésta no es una destrucción efectiva o real del objeto, sino una destrucción potencial, ya que precisamente es la sobrevivencia del objeto la que permite el pasaje de la relación al uso. Pero entonces ¿cómo debe entenderse la destrucción? La actividad destructiva del sujeto está directamente vinculada con el ejercicio de su agresividad. Sin embargo, Winnicott señala que no debemos entender que en su origen la agresividad corresponda a sentimientos tales como la ira, que son sentimientos mucho más elaborados, sino a la motilidad primaria del bebé.

O sea que agresividad es sinónimo de actividad. En su opinión los impulsos fetales traen consigo un descubrimiento del ambiente. El ambiente sería la oposición con que se tropieza en el mo- 
vimiento, de modo que aquél es experimentado durante el movimiento. Esta observación parece clara. Si el bebé careciera de motilidad, si no pudiera ejecutar movimientos, no tendría forma de encontrar resistencias a los mismos. $\mathrm{Y}$ si no puede encontrar resistencias no tiene experiencia de una fuerza exterior a sí mismo. Si bastara mi decisión de mover la mesa para que ésta se mueva, como en efecto sucede con mi brazo, no tendría forma de distinguir que la mesa, a diferencia de mi brazo, no es parte de mí. De este modo, según Winnicott, la oposición con que tropieza el movimiento permite un reconocimiento temprano de un mundo no-yo.

Teniendo en cuenta que la motilidad es la forma primaria de la agresión, veamos cómo juega ésta en la conformación del principio de realidad. El enfoque tradicional hace jugar a la realidad un papel fundamentalmente frustrador en la conformación del principio de realidad. La realidad es lo que meramente se opone a la satisfacción alucinatoria de deseos y será a partir de esa frustración que será necesario conformar un criterio de realidad, a través de la identidad de percepción, para que se autorice la descarga adecuada a la acción específica ${ }^{4}$. A partir de este planteo, la agresividad es reactiva a la experiencia de frustración. En Winnicott se ha producido una inversión, la agresividad no es reactiva al choque con la

4 Cf. Freud (1895). realidad sino que en tanto que motilidad es previa a ésta. Será por lo tanto la agresividad la que permita ir estableciendo la diferencia objetivo-subjetivo y no a la inversa. Pero aquí hay otro punto que destacar y que se vincula con una de las dicotomías que señalamos más arriba. Frente al énfasis puesto por la concepción tradicional en el carácter frustrador de la realidad, Winnicott enfatiza el alivio y la satisfacción que la realidad proveerá frente a los efectos aberrantes de la fantasía sin frenos. Pero este énfasis puesto en el alivio y la satisfacción otorga a la realidad un papel mucho más central en el desarrollo evolutivo del bebé 5 .

Ahora bien, el objeto subjetivo se constituye a partir del encuentro de la creación del pecho por el bebé y el aporte efectivo del pecho por la madre. La repetición de estas experiencias va creando la ilusión de un objeto interno que es parte del yo (identificación primaria. Aspectos femeninos del sujeto). Sin embargo una madre lo bastante buena debe ser capaz también de desilusionar al bebé. La desilusión se produce introduciendo un tiempo entre la

5 "He tratado de desarrollar el tema de que lo que, tanto Freud como Klein eludían de ese modo, era el de las consecuencias de la dependencia y, por tanto, del factor ambiental. Si en verdad la dependencia significa eso, dependencia, la historia de un bebé no se puede escribir en términos de él solamente. Hay que describirla además en términos del ofrecimiento de un ambiente que satisface las necesidades de dependencia o no logra satisfacerlas" (Winnicott, 1971; p. 100). 
alucinación del pecho por el niño y la provisión de éste por parte de la madre. Se darían así momentos en que el pecho es alucinado y la satisfacción no se presenta. Pero el bebé no sabe de ausencias, la presencia real del pecho es un conjunto de sensaciones placenteras y la ausencia real del pecho es un conjunto de sensaciones displacenteras. En tanto que no se distingue todavía entre la sensación y el objeto, en ambos casos estamos ante un objeto, y, asímismo, en ambos casos hay sensaciones. Sin embargo la presencia de las sensaciones displacenteras es distinta de la de las sensaciones placenteras, dando lugar a la presencia de un objeto subjetivo diferente: el objeto malo.

De esta manera, la ausencia del pecho real no es vivida como la ausencia del objeto subjetivo bueno, sino como la presencia del objeto subjetivo malo ${ }^{6}$. Y este objeto subjetivo malo se impone al bebé aun cuando él no lo desee. Vemos con ello que en la constitución del objeto subjetivo malo se han producido dos de los cambios que se proponen para el pasaje de la relación al uso. En tanto que el objeto subjetivo bueno cae bajo el dominio del control omnipotente, es un objeto no-yo. Sin embargo el resto de los cambios aún no se han presentado. El objeto malo sigue siendo un manojo de proyecciones y teniendo un carácter discontinuo. Estas

6 Cf. Berquez, G., "Conceptualización psicoanalítica del Self” en Dorey et al. (1991). otras dos características recién se van a lograr cuando se pueda dar el pasaje de la presencia del objeto subjetivo malo a la ausencia del objeto objetivo. Es aquí donde se debe dar el paso por la destrucción del objeto y la supervivencia del mismo a dicha destrucción. Tal vez este pasaje pueda aclararse por el proceso de desilusión. Como vimos, una madre lo bastante buena introduce una demora entre la alucinación del pecho por el niño y su presentación. Es en esa espera que se conforma el objeto malo con el correspondiente desarrollo de la agresividad. Sin embargo, la madre lo bastante buena presenta el pecho al bebé antes de que éste caiga en una angustia inimaginable. Si el pecho no es aportado en el momento adecuado, la tensión en el bebé aumenta y las sensaciones displacenteras son vividas relativamente por el niño como ataques del objeto malo. En ese caso habría una efectiva destrucción del objeto, ya que, como señala Winnicott, que el objeto haya sido destruido no significa que no tome represalias. Sin embargo, si el pecho es presentado al bebé con una dilación adecuada, la aceptación de éste por el bebé calma sus ansiedades de haberlo destruido.

La repetición de este proceso daría lugar a la secuencia narrada por Winnicott: Después que el sujeto "se relaciona con el objeto" (alucina el pecho, objeto subjetivo) viene "el sujeto destruye al objeto" (dilación introducida por la madre, objeto subjetivo malo, 
fantasía de destrucción del objeto); y después puede venir "el objeto sobrevive a la destrucción por el sujeto" (la madre aporta el pecho con la dilación adecuada $)^{7}$.

La repetición de esta secuencia permite que el objeto vaya perdiendo las características de ser un haz de proyecciones y adquiera una continuidad. Continuidad que podría entenderse como la supervivencia del objeto en los momentos en que es destruido en la fantasía. Podríamos suponer que una vez que la madre instaura la dilación a la que nos estamos refiriendo y finalmente provee del pecho al niño, el estado de éste no es el de la creatividad primaria, previo a esa dilación. El pecho se presenta aun cuando el niño no lo ha creado.

De este modo, una vez que este proceso se ha operado, el niño está en condiciones de "relacionarse" con el objeto en la modalidad del uso de objeto.
7 Cf. Winnicott (1971). Las expresiones entre paréntesis son nuestras.

\section{REFERENCIAS}

Berquez, G. (1991). Conceptualización psicoanalítica del Self. En R. Dorey et al., L'Inconscient et la science. Paris: Dunod.

Davis, M. \& Wallbridge, D. (1988). Límite y Espacio: Introducción a la obra de D.W. Winnicott. Buenos Aires: Amorrortu.

Freud, S. (1895). Proyecto de Psicología. En Obras Completas de Sigmund Freud (Vol. 1. pp. 323-436). Buenos Aires: Amorrortu.

Winnicott, W.D. (1971). Playing and Reality. London: Travistock Publications. 